\title{
An Infant with Liver, Kidney, Skin and Musculoskeletal Abnormalities
}

\author{
Hasosah $\mathrm{M}^{* 1}$, Algabsani $\mathrm{H}^{2}$, Alsharif $\mathrm{E}^{3}$ and Alsharif $\mathrm{A}^{3}$ \\ ${ }^{1}$ Departments of Pediatric Gastroenterology, King Abdul-Aziz Medical City, National Guard Hospital, Jeddah, Saudi \\ Arabia \\ ${ }^{2}$ Departments of Pediatric, King Abdul-Aziz Medical City, National Guard Hospital, Jeddah, Saudi Arabia \\ ${ }^{3}$ Departments of Family Medicine, King Abdul-Aziz Medical City, National Guard Hospital, Jeddah, Saudi Arabia
}

*Corresponding author: Hasosah M, MD, Associated Professor of Pediatrics, Consultant Pediatric Gastroenterologist, King Saud bin Abdulaziz University for Health Sciences, King Abdullah International Medical Research Center, National Guard Hospital, P O BOX 8202, Jeddah 21482, Saudi Arabia, Tel: 00966550105599, E-mail: hasosah2007@yahoo.com

Citation: Hasosah M, Algabsani H, Alsharif E, Alsharif A (2018) An Infant with Liver, Kidney, Skin and Musculoskeletal Abnormalities. J Case Rep Stud 6(1): 104. doi: 10.15744/2348-9820.6.104

Received Date: February 26, 2018 Accepted Date: February 26, 2018 Published Date: February 28, 2018

\section{Case Report}

A 21 day-old boy infant was admitted to pediatric intensive care unit with hypotonia, jaundice and abdominal distention. He was born at $37^{\text {th }}$ week of gestation from first cousin Saudi parents via cesarian section with a birth weight of $2200 \mathrm{~g}$. Physical examination revealed the weight, the length was and the head circumference were $<3^{\text {rd }}$ percentile. Dysmorphic features included flattened nasal bridge, high arched palate, lax skin and micrognathia. There were generalized hypotonia, hepatomegaly, abdominal distention and multiple contractures such as radial deviation of the wrist joint (Figure 1). Laboratory investigations revealed hemoglobin was $16.6 \mathrm{~g} / \mathrm{dl}$, leucocytes: $14.9 / \mathrm{mm}^{3}$, platelets: $83,000 / \mathrm{mm}^{3}$, serum total bilirubin level: $13.5 \mathrm{mg} / \mathrm{dl}$, conjugated bilirubin level: $4.3 \mathrm{mg} / \mathrm{dl}$, aspartate aminotransferase (AST): $555 \mathrm{U} / \mathrm{L}$, alkaline phosphatase (ALP): $960 \mathrm{U} / \mathrm{L}$. Aminoacid analyses of blood and urine, tandem mass were all within normal limits. He was diagnosed as renal tubulopathy based on polyuria with proteinuria: $167 \mathrm{mg} / \mathrm{dL}$, elevated fractional excretion of $\mathrm{Na}$ (FENA: 4.89\%) and fractional excretion of K (FEK: 66\%). Abdominal ultrasound revealed hepatomegaly and bilateral mildly increased renal echogenity. Whole exome sequencing (WES) was performed and showed mutations in the VPS33B gene. Based on all signs and laboratory findings, the baby was diagnosed as arthrogryposis, renal dysfunction, and cholestasis (ARC) syndrome.

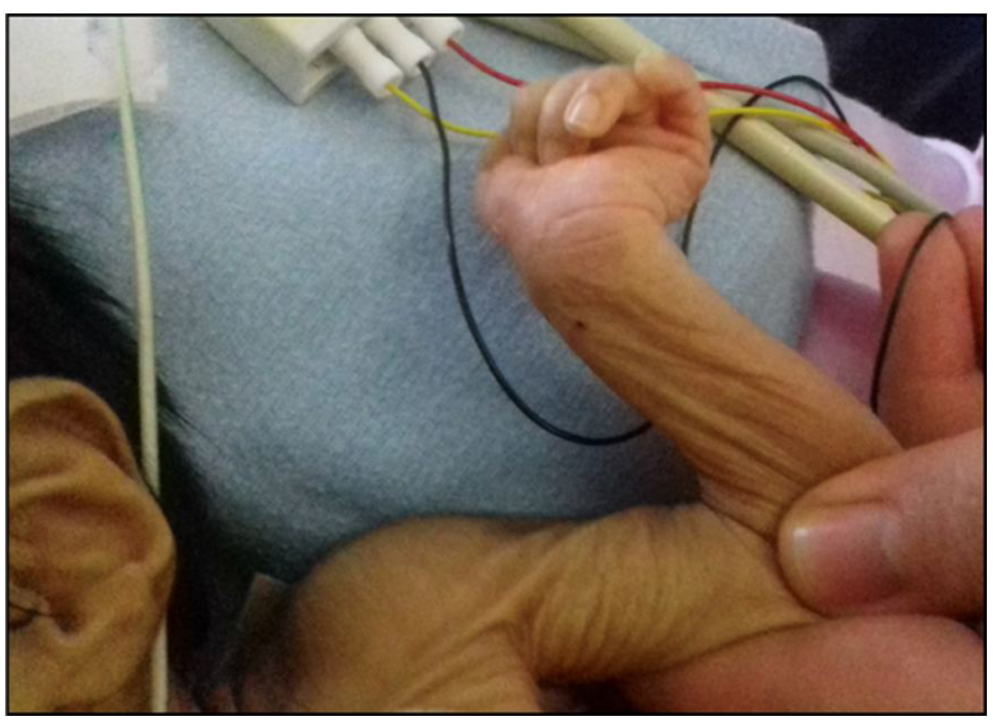

Figure 1: Appearance of ARC syndrome. Lax skin, radial deviation and contracture of the wrist joint

Keywords: Arthrogryposis; Renal dysfunction; Cholestasis

Arthrogryposis, renal dysfunction, and cholestasis (ARC) syndrome is an autosomal recessive disorder caused by mutations in the VPS33B gene [1]. 
This syndrome mainly effects liver, kidney, and skin, central nervous and musculoskeletal systems.

The first component of ARC syndrome is arthrogryposis multiplex congenital [2]. The most common anomalies of musculoskeletal system in ARC syndrome are muscle atrophy, radial deviation of the wrist and flexion contracture of the knee. Osteopenia and pathological fractures can be observed in this syndrome owing to reduced reabsorption of phosphate ions via renal tubules and secondary to hyperparathyroidism [2].

The second component ARC syndrome is cholestatic jaundice and hepatomegaly [3]. These are the most common symptoms in ARC syndrome. Normal GGT levels, mildly elevated AST and ALT levels without biliary obstruction have been described in all patients who have ARC syndrome [3]. Liver histology in the patients with ARC syndrome suggests paucity of bile ducts, lipofuscin deposition and giant cell hepatitis [4].

The third component of ARC syndrome is renal tubular dysfunction which is characterized by multiple features of renal Fanconi syndrome including glucosuria, phosphaturia, generalized aminoaciduria and renal tubular acidosis [5]. Renal tubular dysfunction may present in the first few days of life or later around the age of two months. Most of the patients also present symptoms of nephrogenic diabetes insipidus and Fanconi syndrome [5].

Additional clinical symptoms of ARC syndrome principally include ichthyosis, abnormal platelet count and function, secondary infection, and cardiovascular anomalies [6].

The prognosis of the syndrome is very poor and most patients die by the age of 7 months because of recurrent infections, severe dehydratation, metabolic acidosis or internal hemorrhaging [7].

No specific treatment for ARC syndrome currently exists; rather, supportive care-including fluid infusion, anti-infection, supplement with ursodeoxycholic acid, fat-soluble vitamins, calcium glubionate, L-thyroxine and phosphate-is administered to patients for improving the quality of life. Nevertheless, some patients with joint contractures, congenital hip dislocation, and vertical talus are in need of immediate orthopedic intervention due to delayed diagnosis. Unfortunately, as curative treatment for ARC syndrome is unavailable, all of the patients die within several months. Therefore, extensive research of family history, classical clinical presentations and genetic mutational analysis should be performed for diagnosing and to initiate the therapy at early stage.

\section{References}

1. Gissen P, Tee L, Johnson CA, Genin E, Caliebe A, et al. (2006) Clinical and molecular genetic features of ARC syndrome. Hum Genet 120: $396-409$.

2. Tekin N, Durmuş-Aydoğdu S, Dinleyici EC, Bör O, Bildirici K, et al. (2005) Clinical and pathological aspects of ARC syndrome in two siblings. Turk J Pediatr 47: 67-70.

3. Wang JS, Zhao J, Li LT (2014) ARC syndrome with high GGTcholestasis caused by VPS33B mutations. World J Gastroenterol 20: 4830-4.

4. Abdullah MA, Al-Hasnan Z, Okamoto E, Abomelha AM (2000) Arthrogryposis, renal dysfunction and cholestasis syndrome. Saudi Med J 21: 297-9.

5. Nili F, Akbari-Asbaghe P, Oloomi-Yazdi Z, Hadjizadeh N, Nayeri F, et al. (2008) Wide spectrum of clinical features in a case of arthrogryposis- renal tubular dysfunction-cholestasis syndrome. Arch Iran Med 11: 569-72.

6. Cullinane AR, Straatman-Iwanowska A, Seo JK, Ko JS, Song KS, et al. (2009) Molecular investigations to improve diagnostic accuracy in patients with ARC syndrome. Hum Mutat 30: E330-7.

7. Laforgia N, Burattini MG, Giannuzzo S, Storelli S, Mautone A (2005) ARC syndrome. Acta Pædiatr 94: 254-5. 